\title{
The Fučik spectrum of the $p$-Laplace equations with different weights and its resonance problems
}

\author{
Wenyong Gan ${ }^{1 *}$, Di Geng ${ }^{2}$ and Jian Chen ${ }^{1}$
}

\section{"Correspondence:}

gan.wyong@aliyun.com

'Department of Mathematics,

Foshan University, Foshan,

Guangdong 528000, P.R. China

Full list of author information is

available at the end of the article

\begin{abstract}
In the present paper, our purpose is to construct the Fučik spectrum $C_{I}^{ \pm}(c f .(2.13)$ and (2.17) below) with different weights for the $p$-Laplacian. As an application, we will discuss the existence of nontrivial solutions to the $p$-Laplace equations with resonance on the Fučik spectrum by making use of variational methods and Morse theory.
\end{abstract}

Keywords: $p$-Laplace; Fučik spectrum; nontrivial solutions; variational methods; critical groups; Morse theory

\section{Introduction}

In this paper, we consider the existence of nontrivial solutions to the Dirichlet boundary value problem

$$
\begin{cases}-\Delta_{p} u=\lambda a(x)\left(u^{+}\right)^{p-1}-\mu b(x)\left(u^{-}\right)^{p-1}+g(x, u), & \text { in } \Omega, \\ u=0, & \text { on } \partial \Omega,\end{cases}
$$

where $\Omega$ is a bounded domain in $\mathbb{R}^{N}$ with smooth boundary $\partial \Omega, \Delta_{p}$ denotes the $p$-Laplace operator, that is, $\Delta_{p} u=\nabla\left(|\nabla u|^{p-2} \nabla u\right), u^{ \pm}=\max \{ \pm u(x), 0\}$, and $a(x), b(x) \in L^{r}(\Omega)$, where $r>\frac{N}{p}$ if $1<p \leq N$ and $r=1$ if $p>N$. We assume that $g: \Omega \times \mathbb{R} \rightarrow \mathbb{R}$ is a Caratheodory function.

It is well known that the value of $(\lambda, \mu)$ plays an important role in the study of the solvability of (1.1). The approach here of course requires the preliminary study of weighted asymmetric eigenvalue problems of the form

$$
\begin{cases}-\Delta_{p} u=\lambda a(x)\left(u^{+}\right)^{p-1}-\mu b(x)\left(u^{-}\right)^{p-1}, & \text { in } \Omega, \\ u=0, & \text { on } \partial \Omega .\end{cases}
$$

The set $\Sigma_{p}(a, b)$, the Fučik spectrum of the $p$-Laplacian with the weights $a(x)$ and $b(x)$ on $W_{0}^{1, p}$, is defined by those $(\lambda, \mu) \in \mathbb{R}^{2}$ such that (1.2) has a nontrivial solution $u$.

The generalized notion of spectrum was introduced for $p=2$ (i.e. in the linear case) in the 1970s by Fučik [1] and Dancer [2] in connection with the study of the so-called

(c) The Author(s) 2017. This article is distributed under the terms of the Creative Commons Attribution 4.0 International License (http://creativecommons.org/licenses/by/4.0/), which permits unrestricted use, distribution, and reproduction in any medium, provided you give appropriate credit to the original author(s) and the source, provide a link to the Creative Commons license, and indicate if changes were made. 
jumping non-linearities. From then on, further work has been done in the study of $\Sigma_{2}(1,1)$; $c f$. [3-5]. The quasilinear case of $p \neq 2$ and $N=1$, in the situation where $a(x)$ and $b(x)$ are nonconstant and different, was investigated in [6-9] (for $a(x)$ and $b(x)>0$ ) and [10] (for $a(x)$ and $b(x)$ are indefinite). From the above papers, $\Sigma_{p}(a, b)$ has the same general shape as in the linear ODE case.

In the quasilinear PDE case, when $\lambda a=\mu b$,

$$
\begin{cases}-\Delta_{p} u=\lambda a(x)|u|^{p-2} u, & \text { in } \Omega, \\ u=0, & \text { on } \partial \Omega .\end{cases}
$$

It is well known that problem (1.3) has an unbound sequence of variational eigenvalues $\lambda_{l}(a)$ satisfying a standard min-max characterization, $\lambda_{l}(a) \rightarrow+\infty(l \rightarrow \infty)$. The first eigenvalue $\lambda_{1}(a)$ of $-\Delta_{p} u$ is positive, simple, and admits a positive eigenfunction $\varphi_{a}$ (see Lindqvist [11] for the case $a(x)=1$ and Cuesta [12] for the case where $a(x)$ is an indefinite weights), where $\lambda_{1}(a):=\min \left\{\int_{\Omega}|\nabla u|^{p}: u \in W_{0}^{1, p}(\Omega)\right.$ and $\left.\int_{\Omega} a|u|^{p}=1\right\}<\lambda_{2}(a):=\min \{\lambda \in$ $\mathbb{R}: \lambda$ is the eigenvalue and $\left.\lambda>\lambda_{1}(a)\right\}$. We denote $e_{a}(x):=\frac{\varphi_{a}}{\left\|\varphi_{a}\right\|_{1, p}}$. According to Arias et al. [13], we know that $\Sigma_{p}(a, b)$ contains the two lines $\lambda_{1}(a) \times \mathbb{R}$ and $\mathbb{R} \times \lambda_{1}(b)$.

As in [13], the first nontrivial eigenvalue which admits a sign-changing eigenfunction of the eigenvalue problem with weights $a(x)$ and $b(x)$ is defined as $c(a, b):=\inf _{\gamma \in \Gamma} \max _{t \in[-1,1]}$ $A(\gamma(t))$, where $A(u):=\int_{\Omega}|\nabla u|^{p} d x, \Gamma:=\left\{\gamma \in C\left([-1,1], \mathscr{M}_{a, b}\right): \gamma(-1)=\varphi_{a}\right.$ and $\left.\gamma(1)=\varphi_{b}\right\}$, and $\mathscr{M}_{a, b}:=\left\{u \in W_{0}^{1, p}(\Omega): \int_{\Omega} a(x)\left(u^{+}\right)^{p}+b(x)\left(u^{-}\right)^{p} d x=1\right\}$. Hence, the first nontrivial curve in $\Sigma_{p}(a, b)$ through $(c(a, b), c(a, b))$ asymptotic to $\lambda_{1}(a) \times \mathbb{R}$ and $\mathbb{R} \times \lambda_{1}(b)$ at infinity was constructed by Cuesta et al. [14] for the case $a=b=1$, and by Arias et al. [13] for the case with two different nonconstant weights. More recently, unbounded curves $\left\{C_{l}^{ \pm}\right\}$in $\Sigma_{p}(a, b)$ have been constructed and variationally characterized by min-max procedures by Micheletti and Pistoia [15] for $p>2, a=b=1$, and by Perera [16] for $p>1, a=b=1$ and [17] for the fractional $p$-Laplace operator.

Related studies can also be found in [18-20].

It is our purpose in this paper to initiate the study of (1.2) and its relation with the solvability of (1.1) in the general case: $a(x), b(x) \geq 0$ are possibly nonconstant and different. Hence we will discuss the existence of the Fučik spectrum for (1.2) besides $\lambda_{1}(a) \times \mathbb{R}$, $\mathbb{R} \times \lambda_{1}(b)$ and $\mathcal{C}:=\{(\alpha(s), s \alpha(s)): \alpha(s)=c(a, s b), s>0\}$ (in [13]), which is an open question raised in [13].

As an application, we will concern ourselves with the resonance type of the problem (1.1). The resonance problem has been studied by Perera [16] and Guo and Liu [21] for the case $(\lambda, \mu) \in \Sigma_{p}(a, b)$, with $a=b=1$. The purpose of this paper is to discuss the general resonance case $(\lambda, \mu) \in \Sigma_{p}(a, b)$, with nonconstant and different weights $a(x)$ and $b(x)$. When $(\lambda, \mu) \in \lambda_{1}(a) \times \mathbb{R}\left((\lambda, \mu) \in \mathbb{R} \times \lambda_{1}(b)\right)$, we obtain the existence of nontrivial solution for (1.1) by the Morse theorem. However, the usual Morse theory supposes that the functional $\Phi(u)$ satisfies the Palais-Smale condition ((P.S.) condition for short), which is not clear in the case where the nonlinear term $g(x, u)$ is asymptotic to $|u|^{p-2} u$ at infinity. To overcome this difficulty, Cerami introduced a weaker compactness condition (see [22]) that allows for more general results; see for example [23]. Fortunately, replacing the usual (P.S.) condition by Cerami's weaker compactness condition $\left(\left(C_{c}\right)\right.$ condition for short), we still have the deformation lemma (see [24]). 


\section{Fučik spectrum with different weights}

In this section, we consider the eigenvalues problem of asymmetric $p$-Laplacian with weights (1.2). It is always assumed that $a(x), b(x) \in L^{r}(\Omega)$ with $r$ as in the introduction, i.e. $r>\frac{N}{p}$ if $1<p \leq N$ and $r=1$ if $p>N$.

As is well known, the associated functional of the problem (1.2) is

$$
I(u)=\int_{\Omega}|\nabla u|^{p}-\int_{\Omega} \lambda a(x)\left(u^{+}\right)^{p}+\mu b(x)\left(u^{-}\right)^{p}, \quad u \in W_{0}^{1, p}(\Omega) .
$$

We introduce two new functionals as follows:

$$
\begin{aligned}
& J_{s, t, s_{0}}^{+}(u)=\int_{\Omega}|\nabla u|^{p}-s a(x)\left(u^{+}\right)^{p}-\left(s_{0}+t\right) b(x)\left(u^{-}\right)^{p}+s_{0} a(x)\left(u^{-}\right)^{p}, \\
& J_{s, t, s_{0}}^{-}(u)=\int_{\Omega}|\nabla u|^{p}-s b(x)\left(u^{-}\right)^{p}-\left(s_{0}+t\right) a(x)\left(u^{+}\right)^{p}+s_{0} b(x)\left(u^{+}\right)^{p},
\end{aligned}
$$

where $u \in W_{0}^{1, p}(\Omega), s_{0}$ is a positive constant to be determined later.

The Finsler manifold we need is defined as follows:

$$
S(a):=\left\{\left.u \in W_{0}^{1, p}(\Omega)\left|\int_{\Omega} a(x)\right| u(x)\right|^{p} d x=1\right\} .
$$

$S(b)$ can be considered in a similar way. We will consider the functionals $J_{s, t, s_{0}}^{+}$on the manifold $S(a)$ and $J_{s, t, s_{0}}^{-}$on the manifold $S(b)$, respectively. Let us just deduce the case $J_{s, t, s_{0}}^{+}$on $S(a)$, since the other case can be done similarly.

For each integer $l \geq 1$, we define maps family $\mathcal{F}_{l}(a)$ as

$$
\mathcal{F}_{l}(a):=\left\{A \subset S(a) \text {; there exists an odd continuous surjection map } h: S^{l-1} \rightarrow A\right\},
$$

where $S^{l-1}$ is the unit sphere in $\mathbb{R}^{l}$.

As observed by Drábek and Robinson [25], we can define an unbounded sequence of functionals $J(u)$ by

$$
\lambda_{l}(a):=\inf _{A \in \mathcal{F}_{l}} \max _{u \in A} J(u)
$$

where $J(u)=\int_{\Omega}|\nabla u| d x=J_{0,0,0}(u)$. It is shown that $\lambda_{l}(a)$ is the $l$ th eigenvalue of $-\Delta_{p}$ with weight $a(x)$, although it is not known whether this gives a complete list of eigenvalues. Furthermore,

$$
\lambda_{l}(a) \rightarrow+\infty, \quad \text { as } l \rightarrow \infty
$$

In the sequel we fix some $l \geq 2$ and suppose that

$$
\lambda_{l-1}(a)<\lambda_{l}(a)
$$

For any $\varepsilon \in\left(0, \lambda_{l}(a)-\lambda_{l-1}(a)\right)$, we can choose an $A^{l-2} \in \mathcal{F}_{l-1}(a)$, such that

$$
\max _{u \in A^{l-2}} J(u)<\lambda_{l-1}(a)+\varepsilon
$$


Let $h_{l-2}: S^{l-2} \rightarrow A^{l-2}$ be any fixed continuous surjection and denote

$$
\begin{aligned}
\mathcal{F}_{l}^{+}(a):= & \left\{A_{+} \subset S(a) ; \text { there is a continuous surjection map } h: S_{+}^{l-1} \rightarrow A,\right. \\
& \text { such that } \left.\left.h\right|_{S^{l-2}}=h_{l-2}\right\},
\end{aligned}
$$

where $S_{+}^{l-1}$ is the upper hemisphere of $S^{l-1}$ with boundary $S^{l-2}$. Then $\mathcal{F}_{l}^{+}(a)$ is a homotopystable family of compact subsets of $S(a)$ with closed boundary $A^{l-2}$, that is,

(i) every set $A_{+} \in \mathcal{F}_{l}^{+}(a)$ contains $A^{l-2}$;

(ii) for any set $A_{+} \in \mathcal{F}_{l}^{+}(a)$ and any deformation $\eta \in C([0,1] \times S(a) ; S(a))$ satisfying $\eta(t, u)=u$ for all $(t, u) \in(\{0\} \times S(a)) \cup\left([0,1] \times A^{l-2}\right)$ we have $\eta(\{1\} \times A) \in \mathcal{F}_{l}^{+}(a)$.

Now, we define

$$
c_{l}^{+}\left(s, t, s_{0}\right):=\inf _{A_{+} \in \mathcal{F}_{l}^{+}(a)} \max _{u \in A_{+}} J_{s, t, s_{0}}^{+}(u) .
$$

Similarly, we can define

$$
c_{l}^{-}\left(s, t, s_{0}\right):=\inf _{A_{-} \in \mathcal{F}_{l}^{-}(b)} \max _{u \in A_{-}} J_{s, t, s_{0}}^{-}(u)
$$

We can make sure that $c_{l}^{ \pm}\left(s, t, s_{0}\right)$ is the critical value of $J_{s, t, s_{0}}^{ \pm}(u)$, under some assumptions. Furthermore, the critical point of $J_{s, t, s_{0}}^{ \pm}(u)$ corresponding to $c_{l}^{ \pm}\left(s, t, s_{0}\right)$ in which $s_{0}$ is determined in a special way, is just the solution to (1.2), that is, we will establish the following result.

Theorem 2.1 Suppose that the weights $a(x)$ and $b(x)$ are nonnegative and, for some fixed constants $k^{\prime} \geq 1 \geq k>0$,

$$
0 \leq k a(x) \leq b(x) \leq k^{\prime} a(x), \quad \text { a.e. } x \in \Omega,
$$

where $k$ and $k^{\prime}$ satisfy

$$
\left(\frac{1}{k}-\frac{1}{k^{\prime}}\right) \lambda_{l}(a)<\left(\lambda_{l}(a)-\lambda_{l-1}(a)\right)(1-\delta),
$$

where $\delta \in(0,1)$. Suppose that $s$ and $t$ are in some intervals as follows:

$$
\begin{aligned}
t \in & \left(\frac{\lambda_{l}(a)}{k}-\left(\lambda_{l}(a)-\lambda_{l-1}(a)\right), \frac{\lambda_{l}(a)}{k^{\prime}}-\delta\left(\lambda_{l}(a)-\lambda_{l-1}(a)\right)\right] ; \\
s \in & \left(\max \left\{\lambda_{l-1}(a),(k-1)\left(\lambda_{l}(a)-\lambda_{l-1}(a)\right)+k^{\prime} t\right\},\right. \\
& \left.\min \left\{k\left(\delta\left(\lambda_{l}(a)-\lambda_{l-1}(a)\right)+t\right), \lambda_{l}(a)-\delta\left(\lambda_{l}(a)-\lambda_{l-1}(a)\right)\right\}\right] .
\end{aligned}
$$

Then, for any $s_{0}$ in $\left[\delta\left(\lambda_{l}(a)-\lambda_{l-1}(a)\right), \lambda_{l}(a)-\lambda_{l-1}(a)\right), c_{l}^{+}\left(s, t, s_{0}\right)$ is a critical value of $J_{s, t, s_{0}}^{+}(u)$ on $S(a)$. In particular, the set of the Fučik spectrum of $-\Delta_{p}$ with weights $a(x)$ and $b(x)$ greater than $c(a(x), b(x))$ includes $\left(s+c_{l}^{+}\left(s, t, s_{0}\right), t+c_{l}^{+}\left(s, t, s_{0}\right)\right)$ for $s$ and $t$ in the above intervals, and some well-defined $s_{0} \in\left[\delta\left(\lambda_{l}(a)-\lambda_{l-1}(a)\right), \lambda_{l}(a)-\lambda_{l-1}(a)\right)$ satisfying

$$
c_{l}^{+}\left(s, t, s_{0}\right)=s_{0} .
$$


Lemma 2.1 $J_{s, t, s_{0}}^{+}(u)$ satisfies the (P.S.) condition on $S(a)$.

Proof Let $\left\{u_{n}\right\} \subset S(a)$ and $t_{n} \in \mathbb{R}$ be sequence, such that, for some constant $k$,

$$
\left|J_{s, t, s_{0}}^{+}\left(u_{n}\right)\right| \leq k
$$

and

$$
\begin{aligned}
& \int_{\Omega}\left\{\left|\nabla u_{n}\right|^{p-2} \nabla u_{n} \nabla w-\left[s a\left(u_{n}^{+}\right)^{p-1}-\left(s_{0}+t\right) b\left(u_{n}^{-}\right)^{p-1}+s_{0} a\left(u_{n}^{-}\right)^{p-1}\right] w\right. \\
& \left.-t_{n} a\left|u_{n}\right|^{p-2} u_{n} w\right\} \leq \varepsilon_{n}\|w\|_{1, p},
\end{aligned}
$$

for all $w \in W_{0}^{1, p}(\Omega)$, where $\varepsilon_{n} \rightarrow 0$, as $n \rightarrow \infty$ and $\|\cdot\|_{1, p}$ represents the norm of $W_{0}^{1, p}(\Omega)$. From (2.7) it follows that $\left\{u_{n}\right\}$ remains bounded in $W_{0}^{1, p}(\Omega)$; consequently, for a subsequence, $u_{n} \rightarrow u$ weakly in $W_{0}^{1, p}(\Omega)$, strongly in $L^{p}(\Omega)$ and almost everywhere in $\Omega$. Putting $w=u_{n}-u$ in equation (2.8), we also see that $t_{n}$ remains bounded. We then have

$$
\begin{aligned}
\int_{\Omega}\left|\nabla u_{n}\right|^{p-2} \nabla u_{n}\left(\nabla u_{n}-u\right)= & \int_{\Omega}\left[s a\left(u_{n}^{+}\right)^{p-1}-\left(s_{0}+t\right) b\left(u_{n}^{-}\right)^{p-1}+s_{0} a\left(u_{n}^{-}\right)^{p-1}\right]\left(u_{n}-u\right) \\
& +\int_{\Omega} t_{n} a\left|u_{n}\right|^{p-2} u_{n}\left(u_{n}-u\right)+o\left(\varepsilon_{n}\right),
\end{aligned}
$$

where the right-hand side goes to zero as $n \rightarrow \infty$, i.e.

$$
\int_{\Omega}\left|\nabla u_{n}\right|^{p-2} \nabla u_{n}\left(\nabla u_{n}-u\right) \rightarrow 0, \quad n \rightarrow \infty .
$$

It is sufficient to obtain $u_{n} \rightarrow u$ strongly in $W_{0}^{1, p}$.

Lemma 2.2 As a function of $s, t, s_{0}$, the min-max value

$$
c_{l}^{+}\left(s, t, s_{0}\right):=\inf _{A_{+} \in \mathcal{F}_{l}^{+}(a)} \max _{u \in A_{+}} J_{s, t, s_{0}}^{+}(u)
$$

is continuous on $s_{0}$.

Proof Let $\left\{s_{n}\right\} \subset\left[\delta\left(\lambda_{l}(a)-\lambda_{l-1}(a)\right), \lambda_{l}(a)-\lambda_{l-1}(a)\right]$ be a sequence such that

$$
s_{n} \rightarrow s_{0}, \quad \text { as } n \rightarrow \infty .
$$

Taking $A_{+} \in \mathcal{F}_{l}^{+}(a)$ in the following equation:

$$
J_{s, t, s_{0}}^{+}(u)-J_{s, t, s_{n}}^{+}(u)=\left(s_{0}-s_{n}\right) \int_{\Omega}(a-b)\left(u^{-}\right)^{p} .
$$

Then we can see that

$$
\begin{aligned}
& \max _{A_{+}} J_{s, t, s_{n}}^{+}(u)-\max _{A_{+}}\left(s_{n}-s_{0}\right) \int_{\Omega}(a-b)\left(u^{-}\right)^{p} \\
& \quad \leq \max _{A_{+}} J_{s, t, s_{0}}^{+}(u) \leq \max _{A_{+}} J_{s, t, s_{n}}^{+}(u)+\max _{A_{+}}\left(s_{0}-s_{n}\right) \int_{\Omega}(a-b)\left(u^{-}\right)^{p} .
\end{aligned}
$$


Noting that $k a(x) \leq b(x) \leq k^{\prime} a(x)$, we have

$$
\begin{aligned}
\max _{A_{+}}\left(s_{n}-s_{0}\right) \int_{\Omega}(a-b)\left(u^{-}\right)^{p} & \leq\left|s_{n}-s_{0}\right|\left(\max \left\{|1-k|,\left|k^{\prime}-1\right|\right\}\right) \max _{A_{+}} \int_{\Omega} a\left(u^{-}\right)^{p} \\
& \leq\left(\max \left\{|1-k|,\left|k^{\prime}-1\right|\right\}\right)\left|s_{n}-s_{0}\right|:=\varepsilon_{n},
\end{aligned}
$$

where $\varepsilon_{n} \rightarrow 0$, as $n \rightarrow \infty$. By (2.9) we have

$$
c_{l}^{+}\left(s, t, s_{n}\right)-\varepsilon_{n} \leq c_{l}^{+}\left(s, t, s_{0}\right) \leq c_{l}^{+}\left(s, t, s_{n}\right)+\varepsilon_{n} .
$$

Letting $n \rightarrow \infty$, we have

$$
\limsup _{n \rightarrow \infty} c_{l}^{+}\left(s, t, s_{n}\right) \leq c_{l}^{+}\left(s, t, s_{0}\right) \leq \liminf _{n \rightarrow \infty} c_{l}^{+}\left(s, t, s_{n}\right)
$$

which shows that $\lim _{n \rightarrow \infty} c_{l}^{+}\left(s, t, s_{n}\right)=c_{l}^{+}\left(s, t, s_{0}\right)$. The proof is completed.

Proof of Theorem 2.1 We first claim that

$$
c_{l}^{+}\left(s, t, s_{0}\right) \geq \lambda_{l}(a)-s .
$$

If it is not the case, that is, $c_{l}^{+}\left(s, t, s_{0}\right)<\lambda_{l}(a)-s$, then we can choose some $A_{+} \in \mathcal{F}_{l}^{+}(a)$, such that

$$
\max _{u \in A_{+}} J_{s, t, s_{0}}^{+}(u)<\lambda_{l}(a)-s
$$

By setting $A=A_{+} \cup\left(-A_{+}\right)$, we get an odd symmetric set $A$. The set $A$ is thus in $\mathcal{F}_{l}(a)$. Since the functional $J(u)=J_{0,0,0}(u)$ is even, from (2.4) it follows that

$$
\begin{aligned}
\lambda_{l}(a) & \leq \max _{A} J(u)=\max _{A_{+}} J(u) \\
& \leq \max _{A_{+}} J_{s, t, s_{0}}^{+}(u)+\max _{A_{+}} \int_{\Omega} s a\left(u^{+}\right)^{p}+\left(s_{0}+t\right) b\left(u^{-}\right)^{p}-s_{0} a\left(u^{-}\right)^{p} \\
& \leq \max _{A_{+}} J_{s, t, s_{0}}^{+}(u)+\max _{A_{+}}\left[\int_{\Omega} s a\left(u^{+}\right)^{p}+\left(\left(t+s_{0}\right) k^{\prime}-s_{0}\right) a\left(u^{-}\right)^{p}\right] \\
& \leq \max _{A_{+}} J_{s, t, s_{0}}^{+}(u)+\max \left\{s, t k^{\prime}+\left(k^{\prime}-1\right) s_{0}\right\} \int_{\Omega} a|u|^{p} \\
& <\lambda_{l}(a)-s+\max \left\{s, t k^{\prime}+\left(k^{\prime}-1\right)\left(\lambda_{l}(a)-\lambda_{l-1}(a)\right)\right\} \int_{\Omega} a|u|^{p} \\
& \leq \lambda_{l}(a),
\end{aligned}
$$

which leads to a contradiction. Thus, (2.10) follows.

The inequality (2.10) is crucial in proving the existence of critical value of $J_{s, t, s_{0}}^{+}(u)$ on the manifold $S(a)$. Since the assumption in the theorem implies that, for $\varepsilon \in\left(0, \lambda_{l}(a)-\right.$ $\left.\lambda_{l-1}(a)-s_{0}\right)$, we have

$$
\begin{aligned}
c_{l}^{+}\left(s, t, s_{0}\right) & \geq \lambda_{l}(a)-s \\
& >\lambda_{l-1}(a)+\varepsilon+s_{0}-s
\end{aligned}
$$




$$
\begin{aligned}
& >\max _{A^{l-2}} J(u)+s_{0}-s \\
& =\max _{A^{l-2}} J(u)+\max _{A^{l-2}}\left[\int_{\Omega} s_{0} a\left(u^{+}\right)^{p}+s_{0} a\left(u^{-}\right)^{p}-s a\left(u^{+}\right)^{p}-s a\left(u^{-}\right)^{p}\right] \\
& \geq \max _{A^{l-2}} J(u)+\max _{A^{l-2}}\left[\int_{\Omega} s_{0} a\left(u^{-}\right)^{p}-s \int_{\Omega} a\left(u^{+}\right)^{p}-\left(s_{0}+t\right) \int_{\Omega} b\left(u^{-}\right)^{p}\right] \\
& \geq \max _{A^{l-2}} J_{s, t, s_{0}}^{+}(u) .
\end{aligned}
$$

By Lemma 2.1, we know that $J_{s, t, s_{0}}^{+}$satisfies the (P.S.) condition on $S(a)$. Then it follows from Theorem 3.2 of Ghoussoub [26] that $c_{l}^{+}\left(s, t, s_{0}\right)$ is a critical value of $J_{s, t, s_{0}}^{+}(u)$, that is, according to the Lagrange multiple rule, there is some $u \in W_{0}^{1, p}(\Omega)$, such that

$$
-\Delta_{p} u-s a\left(u^{+}\right)^{p-1}+\left(s_{0}+t\right) b\left(u^{-}\right)^{p-1}-s_{0} a\left(u^{-}\right)^{p-1}=c_{l}^{+}\left(s, t, s_{0}\right)\left[a\left(u^{+}\right)^{p-1}-a\left(u^{-}\right)^{p-1}\right] .
$$

The min-max value $c_{l}^{+}\left(s, t, s_{0}\right)$ is also a function of $s_{0}$, and from Lemma 2.2, we know that $c_{l}^{+}\left(s, t, s_{0}\right)$ is continuous on $s_{0}$. Set

$$
\psi\left(s_{0}\right)=c_{l}^{+}\left(s, t, s_{0}\right)-s_{0}
$$

Next we claim that

$$
\psi\left(\delta\left(\lambda_{l}(a)-\lambda_{l-1}(a)\right)\right) \geq 0 \quad \text { and } \quad \psi\left(\lambda_{l}(a)-\lambda_{l-1}(a)\right) \leq 0 .
$$

In fact, for every $A_{+} \in \mathcal{F}_{l}^{+}(a)$, since $A=A_{+} \cup\left(-A_{+}\right) \in \mathcal{F}_{l}(a)$, on $A$, we have

$$
J(u)=\int_{\Omega}|\nabla u|^{p} \geq \lambda_{l}(a) \int_{\Omega} a|u|^{p} .
$$

Hence, we can estimate the maximum of $J_{s, t, \delta\left(\lambda_{l}(a)-\lambda_{l-1}(a)\right)}(u)$ on $A_{+}$as follows:

$$
\begin{aligned}
\max _{A_{+}} J_{s, t, \delta\left(\lambda_{l}(a)-\lambda_{l-1}(a)\right)}(u) & \\
= & \max _{A_{+}}\left\{\int_{\Omega}|\nabla u|^{p}-s a\left(u^{+}\right)^{p}-\left(t+\delta\left(\lambda_{l}(a)-\lambda_{l-1}(a)\right)\right) b\left(u^{-}\right)^{p}\right. \\
& \left.+\delta\left(\lambda_{l}(a)-\lambda_{l-1}(a)\right) \int_{\Omega} a\left(u^{-}\right)^{p}\right\} \\
\geq & \max _{A_{+}}\left\{\lambda_{l}(a) \int_{\Omega} a|u|^{p}-s a\left(u^{+}\right)^{p}-\left(t+\delta\left(\lambda_{l}(a)-\lambda_{l-1}(a)\right)\right) b\left(u^{-}\right)^{p}\right. \\
& \left.+\delta\left(\lambda_{l}(a)-\lambda_{l-1}(a)\right) \int_{\Omega} a\left(u^{-}\right)^{p}\right\} \\
\geq & \max _{A_{+}}\left\{\lambda_{l}(a) \int_{\Omega} a|u|^{p}\right. \\
& \left.-\max \left\{s, k^{\prime}\left(t+\delta\left(\lambda_{l}(a)-\lambda_{l-1}(a)\right)\right)-\delta\left(\lambda_{l}(a)-\lambda_{l-1}(a)\right)\right\} \int_{\Omega} a|u|^{p}\right\} \\
= & \lambda_{l}(a)-\max \left\{s, k^{\prime}\left(t+\delta\left(\lambda_{l}(a)-\lambda_{l-1}(a)\right)\right)-\delta\left(\lambda_{l}(a)-\lambda_{l-1}(a)\right)\right\} .
\end{aligned}
$$


By the conditions (2.5) and (2.6), we have

$$
\left\{\begin{array}{l}
\lambda_{l}(a) \geq s+\delta\left(\lambda_{l}(a)-\lambda_{l-1}(a)\right) \\
\lambda_{l}(a) \geq k^{\prime}\left(t+\delta\left(\lambda_{l}(a)-\lambda_{l-1}(a)\right)\right) .
\end{array}\right.
$$

Then we see that $\psi\left(\delta\left(\lambda_{l}(a)-\lambda_{l-1}(a)\right)\right) \geq 0$.

Moreover, for any $\varepsilon>0$, we pick up an $A \in \mathcal{F}_{l}(a)$, such that

$$
A=h\left(S^{l-1}(a)\right),\left.\quad h\right|_{S^{l-2}(a)}=h_{l-2},
$$

and

$$
\max _{u \in A} J(u)<\lambda_{l}(a)+\varepsilon .
$$

Denote $A_{+}=h\left(S_{+}^{l-1}(a)\right)$, and then $A_{+} \in \mathcal{F}_{l}^{+}(a)$. For every $u \in A_{+}$,

$$
\begin{aligned}
J_{s, t, \lambda_{l}(a)-\lambda_{l-1}(a)}(u) & \\
= & \int_{\Omega}|\nabla u|^{p}-s a\left(u^{+}\right)^{p}-\left(\lambda_{l}(a)-\lambda_{l-1}(a)+t\right) b\left(u^{-}\right)^{p}+\left(\lambda_{l}(a)-\lambda_{l-1}(a)\right) a\left(u^{-}\right)^{p} \\
\leq & \lambda_{l}(a) \int_{\Omega} a|u|^{p}+\varepsilon \\
& +\int_{\Omega}\left[-s a\left(u^{+}\right)^{p}-\left(\lambda_{l}(a)-\lambda_{l-1}(a)+t\right) b\left(u^{-}\right)^{p}+\left(\lambda_{l}(a)-\lambda_{l-1}(a)\right) a\left(u^{-}\right)^{p}\right] \\
\leq & \left(\lambda_{l}(a)-s\right) \int_{\Omega} a\left(u^{+}\right)^{p}+\varepsilon \\
& +\left[2 \lambda_{l}(a)-\left(\lambda_{l}(a)-\lambda_{l-1}(a)+t\right) k-\lambda_{l-1}(a)\right] \int_{\Omega} a\left(u^{-}\right)^{p} \\
\leq & \max \left\{\lambda_{l}(a)-s, 2 \lambda_{l}(a)-\left(\lambda_{l}(a)-\lambda_{l-1}(a)+t\right) k-\lambda_{l-1}(a)\right\}+\varepsilon .
\end{aligned}
$$

We can deduce that $\psi\left(\lambda_{l}(a)-\lambda_{l-1}(a)\right) \leq 0$. Hence there exists some $s_{0} \in\left[\delta\left(\lambda_{l}(a)-\right.\right.$ $\left.\left.\lambda_{l-1}(a)\right), \lambda_{l}(a)-\lambda_{l-1}(a)\right)$, such that

$$
\psi\left(s_{0}\right)=0
$$

which means that if we take $s_{0}$ in $J_{s, t, s_{0}}(u)$ as above, then

$$
s_{0}=c_{l}^{+}\left(s, t, s_{0}\right) \text {. }
$$

We see that there exists some $u$ which is also the solution, in the weak sense, to the following equation:

$$
-\Delta_{p} u=\left(s+c_{l}^{+}\left(s, t, s_{0}\right)\right) a\left(u^{+}\right)^{p-1}-\left(t+c_{l}^{+}\left(s, t, s_{0}\right)\right) b\left(u^{-}\right)^{p-1} .
$$

Clearly,

$$
C_{l}^{+}:=\left\{\left(s+c_{l}^{+}\left(s, t, s_{0}\right), t+c_{l}^{+}\left(s, t, s_{0}\right)\right): s_{0}=c_{l}^{+}\left(s, t, s_{0}\right)\right\} \subset \Sigma_{p}(a, b)
$$

where $s, t, s_{0}$ and $c_{l}^{+}\left(s, t, s_{0}\right)$ are as in Theorem 2.1. 
In a similar way, suppose $\lambda_{l-1}(b)<\lambda_{l}(b)$ for some fix $l \geq 2$, and then we can get a corresponding conclusion as follows.

Theorem 2.2 Suppose that the weights $a(x)$ and $b(x)$ are nonnegative and, for some fixed constants $k^{\prime} \geq 1 \geq k>0$,

$$
0 \leq k b(x) \leq a(x) \leq k^{\prime} b(x), \quad \text { a.e. } x \in \Omega,
$$

where $k$ and $k^{\prime}$ satisfy

$$
\left(\frac{1}{k}-\frac{1}{k^{\prime}}\right) \lambda_{l}(b)<\left(\lambda_{l}(b)-\lambda_{l-1}(b)\right)(1-\delta),
$$

where $\delta \in(0,1)$. Suppose that $s$ and $t$ are in some intervals as follows:

$$
\begin{aligned}
t \in & \left(\frac{\lambda_{l}(b)}{k}-\left(\lambda_{l}(b)-\lambda_{l-1}(b)\right), \frac{\lambda_{l}(b)}{k^{\prime}}-\delta\left(\lambda_{l}(b)-\lambda_{l-1}(b)\right)\right] ; \\
s \in & \left(\max \left\{\lambda_{l-1}(b),(k-1)\left(\lambda_{l}(b)-\lambda_{l-1}(b)\right)+k^{\prime} t\right\},\right. \\
& \left.\min \left\{k\left(\delta\left(\lambda_{l}(b)-\lambda_{l-1}(b)\right)+t\right), \lambda_{l}(b)-\delta\left(\lambda_{l}(b)-\lambda_{l-1}(b)\right)\right\}\right] .
\end{aligned}
$$

Then, for any $s_{0}$ in $\left[\delta\left(\lambda_{l}(b)-\lambda_{l-1}(b)\right), \lambda_{l}(b)-\lambda_{l-1}(b)\right), c_{l}^{-}\left(s, t, s_{0}\right)$ is a critical value of $J_{s, t, s_{0}}^{-}(u)$ on $S(b)$. In particular, the set of the Fučik spectrum of $-\Delta_{p}$ with weights $a(x)$ and $b(x)$ greater than $c(a(x), b(x))$ includes $\left(t+c_{l}^{-}\left(s, t, s_{0}\right), s+c_{l}^{-}\left(s, t, s_{0}\right)\right)$ for $s$ and $t$ in the above intervals, and some well-defined $s_{0} \in\left[\delta\left(\lambda_{l}(b)-\lambda_{l-1}(b)\right), \lambda_{l}(b)-\lambda_{l-1}(b)\right)$ satisfies

$$
c_{l}^{-}\left(s, t, s_{0}\right)=s_{0} .
$$

Hence,

$$
C_{l}^{-}:=\left\{\left(t+c_{l}^{-}\left(s, t, s_{0}\right), s+c_{l}^{-}\left(s, t, s_{0}\right)\right): s_{0}=c_{l}^{-}\left(s, t, s_{0}\right)\right\} \subset \Sigma_{p}(a, b),
$$

where $s, t, s_{0}$ and $c_{l}^{-}\left(s, t, s_{0}\right)$ are as in Theorem 2.2.

Remark 2.1 Theorem 2.1 and Theorem 2.2 contain the results in [16], in which $a(x)=$ $b(x)=1$; so our results must be much more generic than those in [16].

Remark 2.2 Theorem 2.1 and Theorem 2.2 answer partly the open question raised in [13]: whether there exists any other Fučik spectrum for (1.2) besides $\lambda_{1}(a) \times \mathbb{R}, \mathbb{R} \times \lambda_{1}(b)$ and $\mathcal{C}:=\{(\alpha(s), s \alpha(s)): \alpha(s)=c(a, s b), s>0\}$.

\section{Resonance problems with respect to the Fučik spectrum}

As an application, we consider the resonance problems for the Dirichlet boundary problem (1.1) of the Fučik spectrum type. The first case: $(\lambda, \mu)$ is in the part of the two lines $\lambda_{1}(a) \times R$ and $R \times \lambda_{1}(b)$, which has been defined in [13]. The second case: $(\lambda, \mu) \in C_{l}^{ \pm}$, which is defined in Section 2; see equations (2.13) and (2.17). Similar to Section 2, we always assumed that $a(x), b(x) \in L^{r}$ with $r$ as in the introduction. We assume that $g: \Omega \times \mathbb{R} \rightarrow \mathbb{R}$ is a Caratheodory function. In order to state our main results, we assume that $g(x, u)$ satisfies the following conditions: 
(f $\left.f_{1}\right) \quad g(x, u) \in C(\bar{\Omega} \times \mathbb{R}, \mathbb{R})$ with $g(x, 0)=0$ and $\lim _{|s| \rightarrow \infty} \frac{g(x, s)}{|s|^{p-1}}=0$ uniformly;

$\left(\mathrm{f}_{2}\right) \quad p G(x, s)-g(x, s) s>0$ a.e. $x \in \Omega, \forall s \neq 0$;

$\left(\mathrm{f}_{3}\right) \lim _{|s| \rightarrow \infty}(g(x, s) s-p G(x, s))=-\infty$;

$\left(f_{4}\right)$ for some $v \in(1, p)$, there are constants $m, a_{m}>0$ s.t. $G(x, u) \geq a_{m}|u|^{v}$ for $|u| \leq m$;

$\left(\mathrm{F}_{1}\right)|g(x, u)| \leq V(x)^{p-q}|u|^{q-1}+W(x)^{p-1}$ with $q \leq p$ and nonnegative $V, W \in L^{p}(\Omega)$;

$\left(\mathrm{F}_{2}\right) \exists \theta \in(0,1)$ and $M>0$ such that $G(x, u)-\theta^{p} G\left(x, \frac{u}{\theta}\right) \leq M$, for $|u| \geq M$.

As is well known, the weak solution to the problem (1.1) is a critical point of

$$
\Phi(u)=\int_{\Omega}|\nabla u|^{p}-\lambda a(x)\left(u^{+}\right)^{p}-\mu b(x)\left(u^{-}\right)^{p}-p G(x, u), \quad \forall u \in W_{0}^{1, p}(\Omega),
$$

where $G(x, t)=\int_{0}^{t} g(x, s) d s$, and then we have the following.

Theorem 3.1 Suppose that conditions $\left(f_{1}\right)-\left(f_{4}\right)$ hold, $a(x), b(x) \geq \epsilon_{0}>0$, a.e. in $\Omega$. Then (1.1) has at least one nontrivial weak solution in $W_{0}^{1, p}(\Omega)$ if one of the following conditions holds:

$\left(f_{5}\right) \quad \lambda=\lambda_{1}(a)$; and $\mu$ satisfies

$$
c(a, a) a(x)>\mu b(x) \quad \text { and } \quad \mu \int_{\Omega} b(x) e_{a}^{p}(x) d x \geq 1
$$

$\left(\mathrm{f}_{6}\right) \quad \mu=\lambda_{1}(b)$; and $\lambda$ satisfies

$$
c(b, b) b(x)>\lambda a(x) \text { and } \lambda \int_{\Omega} a(x) e_{b}^{p}(x) d x \geq 1 .
$$

Theorem 3.2 Suppose that $\left(\mathrm{F}_{1}\right),\left(\mathrm{f}_{3}\right)$ and $\left(\mathrm{f}_{5}\right)$ hold, $a(x), b(x) \geq \epsilon_{0}>0$, a.e. in $\Omega$ and satisfy the conditions mentioned in Theorem 2.1 (or in Theorem 2.2), and then the problem (1.1) has a nontrivial solution if $(\lambda, \mu) \in C_{l}^{+}\left(\right.$or $\left.(\lambda, \mu) \in C_{l}^{-}\right)$.

In order to prove Theorem 3.1, we need to state the following conclusions about critical groups. Firstly, we introduce some notations. Let $E:=W_{0}^{1, p}(\Omega), E^{*}$ represent the dual space to $E$, and $J$ be a $C^{1}(E, \mathbb{R})$. Then the critical set of $J$ on $E$ is defined as

$$
K=\left\{u \in E, J^{\prime}(u)=\theta\right\} .
$$

For $c \in \mathbb{R} \cup\{+\infty\}$, we use

$$
J^{c}=\{u \in E, J(u) \leq c\}, \quad J^{c}=\{u \in E, J(u)<c\}
$$

standing for the closed and open sublevel sets of functional $J$ on $E$. Recall that the critical groups of $J$ at its isolated point $u$ with a critical value $c=J(u)$ are defined by

$$
C_{q}(J, u)=H_{q}\left(J^{c} \cap U,\left(J^{c} \cap U\right) \backslash\{u\}\right), \quad \text { for all } q \geq 0,
$$

where $U$ is an isolated neighborhood of $u$, and $H_{q}(\cdot, \cdot)$ is the $q$ th singular relative homology groups with the integer coefficients $\mathbb{Z}$. Moreover, it is well known that $C_{q}(J, u)$ is independent of the choice of $U$ and due to the excision property of singular homology theory. 
Suppose that $-\infty<\inf J(K)$, and we choose $c<\inf J(K)$. The critical groups of $J$ at infinity are defined by

$$
C_{q}(J, \infty)=H_{q}\left(E, J^{c}\right), \quad \text { for all } q \geq 0 .
$$

From the deformation lemma, we see that $C_{q}(J, \infty)$ is independent of the choice of $c<$ $\inf J(K)$ while $J$ satisfies the (P.S.) condition. For details of the topological notation mentioned here and throughout this paper refer to [27].

Lemma 3.1 Suppose that conditions $\left(\mathrm{f}_{1}\right)-\left(\mathrm{f}_{3}\right)$ hold and $\lambda=\lambda_{1}(a)\left(\right.$ or $\left.\mu=\lambda_{1}(b)\right)$; then the functional $\Phi$ satisfies $\left(C_{c}\right)$ condition for all $c \in \mathbb{R}$.

Proof We just give the proof of the case when $\lambda=\lambda_{1}(a)$, and the other case of $\mu=\lambda_{1}(b)$ can be treated similarly.

Suppose that $\left\{u_{n}\right\} \in W_{0}^{1, p}$ is a sequence such that

$$
\begin{aligned}
& \Phi\left(u_{n}\right) \rightarrow c, \\
& \left(1+\left\|u_{n}\right\|_{1, p}\right)\left\|\Phi^{\prime}\left(u_{n}\right)\right\|_{E^{*}} \rightarrow 0 .
\end{aligned}
$$

It is sufficient to obtain that $\left\{u_{n}\right\}$ is bounded. Assume the contrary, that is, $\left\|u_{n}\right\| \rightarrow \infty$. Let $v_{n}=\frac{u_{n}}{\left\|u_{n}\right\|_{1, p}}$. Then, up to a subsequence, we still denote the subsequence by $\left\{v_{n}\right\}$. There is a $v_{0}$ such that $\left\{v_{n}\right\}$ converges weakly to $v_{0}$ in $W_{0}^{1, p}(\Omega), v_{n} \rightarrow v_{0}$ strongly in $L^{p}(\Omega)$, and $v_{n}(x) \rightarrow v_{0}(x)$ almost everywhere in $\Omega$. In (3.3) divided by $\left\|u_{n}\right\|_{1, p}^{p-1}$, and then for every $w \in W_{0}^{1, p}$, we have

$$
\int_{\Omega}\left[\left|\nabla v_{n}\right|^{p-2} \nabla v_{n} \nabla w-\lambda a(x)\left(v_{n}^{+}\right)^{p-1} w+\mu b(x)\left(v_{n}^{-}\right)^{p-1} w-\frac{g\left(x, u_{n}\right) w}{\left\|u_{n}\right\|_{1, p}^{p-1}}\right] d x \rightarrow 0 .
$$

In (3.4), first we claim that $\left\|u_{n}\right\|_{1, p}^{1-p} \int_{\Omega} g\left(x, u_{n}\right) w d x \rightarrow 0$. Indeed, it follows from $\left(\mathrm{f}_{1}\right)$ that, for any $\epsilon>0$, there exists $C_{\epsilon}>0$ such that

$$
|g(x, u)| \leq C_{\epsilon}+\epsilon|u|^{p-1}, \quad \forall(x, u) \in \bar{\Omega} \times \mathbb{R} .
$$

By Hölder's inequality and Sobolev's inequality, we get

$$
\left|\int_{\Omega} \frac{g\left(x, u_{n}\right) w}{\left\|u_{n}\right\|_{1, p}^{p-1}} d x\right| \leq \int_{\Omega} \frac{C_{\epsilon}+\epsilon\left|u_{n}\right|^{p-1}}{\left\|u_{n}\right\|_{1, p}^{p-1}}|w| d x \leq\left(\frac{C_{1}}{\left\|u_{n}\right\|_{1, p}^{p-1}}+\epsilon C_{2}\right)\|w\| \rightarrow 0, \quad n \rightarrow \infty .
$$

Hence from (3.4) we have

$$
\int_{\Omega}\left[\left|\nabla v_{n}\right|^{p-2} \nabla v_{n} \nabla w-\lambda a(x)\left(v_{n}^{+}\right)^{p-1} w+\mu b(x)\left(v_{n}^{-}\right)^{p-1} w\right] d x \rightarrow 0, \quad n \rightarrow \infty .
$$

Putting $w=v_{n}-v_{0}$, it is sufficient to obtain

$$
\int_{\Omega}\left|\nabla v_{n}\right|^{p-2} \nabla v_{n} \nabla\left(v_{n}-v_{0}\right) d x \rightarrow 0, \quad n \rightarrow \infty
$$


And consequently

$$
\int_{\Omega}\left(\left|\nabla v_{n}\right|^{p-2} \nabla v_{n}-\left|\nabla v_{0}\right|^{p-2} \nabla v_{0}\right) \nabla\left(v_{n}-v_{0}\right) d x \rightarrow 0, \quad n \rightarrow \infty
$$

Using the inequality

$$
|\xi-\eta|^{p} \leq c\left[\left(|\xi|^{p-2} \xi-|\eta|^{p-2} \eta\right)(\xi-\eta)\right]^{s / 2}\left[|\xi|^{p}+|\eta|^{p}\right]^{1-s / 2}
$$

where $\xi, \eta \in \mathbb{R}^{N}, c=c(p)>0$, and $s=2$ if $p \geq 2, s=p$ if $1<p<2$, one easily obtains $v_{n} \rightarrow v_{0}$ in $W_{0}^{1, p}$ with $\left\|v_{0}\right\|_{1, p}=1$.

Hence from (3.5) we see that

$$
\int_{\Omega}\left|\nabla v_{0}\right|^{p-2} \nabla v_{0} \nabla w=\int_{\Omega}\left[\lambda a(x)\left(v_{0}^{+}\right)^{p-1} w+\mu b(x)\left(v_{0}^{-}\right)^{p-1} w\right] d x, \quad \forall w \in W_{0}^{1, p} .
$$

Taking $w=v_{0}^{ \pm}$, respectively, we get

$$
\int_{\Omega}\left|\nabla v_{0}^{+}\right|^{p}=\lambda_{1}(a) \int_{\Omega} a(x)\left|v_{0}^{+}\right|^{p}
$$

and

$$
\int_{\Omega}\left|\nabla v_{0}^{-}\right|^{p}=\mu \int_{\Omega} b(x)\left|v_{0}^{-}\right|^{p}
$$

respectively. From the above equality (3.7), we know either $v_{0}^{+}=0$ or $v_{0}=e_{a}$, where $e_{a}$ is the eigenfunction corresponding to $\lambda=\lambda_{1}(a)$. If $v_{0}=e_{a}$, then $u_{n}(x)=\left\|u_{n}\right\|_{1, p} e_{a} \rightarrow+\infty$ for almost everywhere in $\Omega$. If $v_{0}^{+}=0$, then we must have $v_{0}=-e_{b}<0$ where $e_{b}$ is the eigenfunction corresponding to $\mu=\lambda_{1}(b)$, since $v_{0} \neq 0$ and equality (3.8), then $u_{n}(x)=$ $-\left\|u_{n}\right\|_{1, p} e_{b} \rightarrow-\infty$ for almost everywhere in $\Omega$.

From assumption $\left(\mathrm{f}_{3}\right)$, we have

$$
\lim _{n \rightarrow \infty}\left(g\left(x, u_{n}\right) u_{n}-p G\left(x, u_{n}\right)\right)=-\infty, \quad \text { a.e. } x \in \Omega \text {, as } n \rightarrow \infty
$$

However, by (3.2) and (3.3), one has

$$
p \Phi\left(u_{n}\right)-\left\langle\Phi^{\prime}\left(u_{n}\right), u_{n}\right\rangle \rightarrow p c, \quad n \rightarrow \infty
$$

which implies that

$$
\int_{\Omega}\left(g\left(x, u_{n}\right) u_{n}-p G\left(x, u_{n}\right)\right) d x \rightarrow p c, \quad \text { as } n \rightarrow \infty
$$

This contradicts (3.9). Thus $\left\{u_{n}\right\}$ is bounded, and the proof completed.

Lemma 3.2 Suppose that conditions $\left(\mathrm{f}_{1}\right)-\left(\mathrm{f}_{3}\right)$ and $\left(\mathrm{f}_{5}\right)\left(\right.$ or $\left.\left(\mathrm{f}_{6}\right)\right)$ hold, $a(x), b(x) \geq \epsilon_{0}>0$, a.e. in $\Omega$. Then we have

$$
C_{1}(\Phi, \infty) \neq 0 \text {. }
$$


Proof We just give the proof of the case $\left(f_{5}\right)$, and the other case $\left(f_{6}\right)$ can be treated in a similar way.

By $\left(f_{1}\right)$, we know that $\Phi$ is of class $C^{1}$ in $W_{0}^{1, p}(\Omega)$. We consider the decomposition of $W_{0}^{1, p}=V \oplus W$, where $V=\operatorname{span}\left\{e_{a}\right\}$ is the one-dimensional eigenspace associated with $\lambda_{1}(a)$, with $e_{a}>0$ in $\Omega$ and $\left\|e_{a}\right\|_{1, p}=1$, and $W:=\left\{w \in W_{0}^{1, p}(\Omega): \int_{\Omega} a(x) w e_{a}^{p-1} d x=0\right\}$ is a complementary subspace of $V$ in $W_{0}^{1, p}(\Omega)$. Therefore (see [28]), there exists $\bar{\lambda}>\lambda_{1}(a)$ such that

$$
\int_{\Omega}|\nabla u|^{p} d x \geq \bar{\lambda} \int_{\Omega} a(x)|u|^{p} d x, \quad \forall u \in W
$$

We claim that $\bar{\lambda}=c(a, a)$, if it is not the case, that is, there exists $u \in W$, such that

$$
\lambda_{1}(a) \int_{\Omega} a(x)|u|^{p}<\int_{\Omega}|\nabla u|^{p}<c(a, a) \int_{\Omega} a(x)|u|^{p} .
$$

Hence there exist $\lambda_{1}(a)<\theta<c(a, a)$ and $u \in W$, such that

$$
\int_{\Omega}|\nabla u|^{p}=\theta \int_{\Omega} a(x)|u|^{p}
$$

Hence, $\theta$ is the eigenvalue of problem (1.3), which contradicts Theorem 11 in [13] stating that problem (1.3) does not admit any eigenvalue in the open interval $\left(\lambda_{1}(a), c(a, a)\right)$. Then we have

$$
\int_{\Omega}|\nabla u|^{p} d x \geq c(a, a) \int_{\Omega} a(x)|u|^{p} d x, \quad \forall u \in W .
$$

On the other hand, $\left(f_{1}\right)$ implies that

$$
\lim _{|s| \rightarrow \infty} \frac{p G(x, s)}{|s|^{p}}=0
$$

And from $\left(\mathrm{f}_{3}\right)$, we obtain: for any $M>0$, there is some $R>0$ such that

$$
g(x, s) s-p G(x, s) \leq-M, \quad|s|>R \text {, a.e. } x \in \Omega .
$$

Integrating the equality

$$
\frac{d}{d x}\left[\frac{G(x, s)}{|s|^{p}}\right]=\frac{g(x, s) s-p G(x, s)}{|s|^{p+1}},
$$

over the interval $[t, T] \subset[R,+\infty)$, we have

$$
\frac{G(x, T)}{|T|^{p}}-\frac{G(x, t)}{|t|^{p}} \leq \frac{M}{p}\left(\frac{1}{T^{p}}-\frac{1}{t^{p}}\right) .
$$

Noting that $\lim _{T \rightarrow \infty} T^{-p} G(x, T)=0$, and letting $T \rightarrow \infty$ in (3.11), we have

$$
G(x, t) \geq \frac{M}{p}, \quad \forall t \geq R \text {, a.e. } x \in \Omega .
$$


Similarly, it is shown that $G(x, t) \geq \frac{M}{p}, \forall t \leq-R$, a.e. $x \in \Omega$. Hence

$$
\lim _{|t| \rightarrow \infty} G(x, t)=+\infty, \quad \text { a.e. } x \in \Omega
$$

Now, letting $v=\xi e_{a} \in V(\xi \in \mathbb{R})$, from the assumption $\left(\mathrm{f}_{5}\right)$ we have

$$
\begin{aligned}
\Phi(v) & =\frac{1}{p} \int_{\Omega}|\nabla v|^{p} d x-\frac{1}{p} \int_{\Omega}\left[\lambda_{1}(a) a(x)\left(v^{+}\right)^{p}+\mu b(x)\left(v^{-}\right)^{p}\right] d x-\int_{\Omega} G(x, v) d x \\
& = \begin{cases}\frac{1}{p} \xi^{p} \int_{\Omega}\left|\nabla e_{a}\right|^{p} d x-\frac{1}{p} \lambda_{1}(a) \xi^{p} \int_{\Omega} a(x) e_{a}^{p} d x-\int_{\Omega} G\left(x, \xi e_{a}\right), & \xi \geq 0, \\
\frac{1}{p}|\xi|^{p} \int_{\Omega}\left|\nabla e_{a}\right|^{p} d x-\frac{1}{p} \mu|\xi|^{p} \int_{\Omega} b(x) e_{a}^{p} d x-\int_{\Omega} G\left(x, \xi e_{a}\right), & \xi<0\end{cases} \\
& = \begin{cases}-\int_{\Omega} G\left(x, \xi e_{a}\right), & \xi \geq 0, \\
\frac{1}{p}|\xi|^{p}\left[1-\mu \int_{\Omega} b(x) e_{a}^{p} d x\right]-\int_{\Omega} G\left(x, \xi e_{a}\right), & \xi<0\end{cases} \\
& \leq-\int_{\Omega} G\left(x, \xi e_{a}\right) .
\end{aligned}
$$

It follows from Lebesgue's theorem that

$$
\Phi(v) \rightarrow-\infty, \quad \text { for } v \in V \quad \text { and } \quad\|v\|_{1, p} \rightarrow \infty
$$

Now we turn to proving

$$
\inf _{W} \Phi=m>-\infty
$$

Indeed, by $\left(\mathrm{f}_{1}\right)$,we obtain that, for any $\epsilon>0$, there exists $C_{\epsilon}>0$ such that

$$
|G(x, u)| \leq C_{\epsilon}+\epsilon|u|^{p}, \quad \forall(x, u) \in \bar{\Omega} \times \mathbb{R} .
$$

By (3.10) and (3.14), we have, for any $w \in W$,

$$
\begin{aligned}
p \Phi(w)= & \int_{\Omega}|\nabla w|^{p} d x-\int_{\Omega}\left[\lambda_{1}(a) a(x)\left(w^{+}\right)^{p}+\mu b(x)\left(w^{-}\right)^{p}+p G(x, w)\right] d x \\
\geq & c(a, a) \int_{\Omega} a(x)|w|^{p} d x-\int_{\Omega}\left[\lambda_{1}(a) a(x)\left(w^{+}\right)^{p}+\mu b(x)\left(w^{-}\right)^{p}+p G(x, w)\right] d x \\
= & \left(c(a, a)-\lambda_{1}(a)\right) \int_{\Omega} a(x)\left(w^{+}\right)^{p} d x \\
& +\int_{\Omega}[c(a, a) a(x)-\mu b(x)]\left(w^{-}\right)^{p} d x-p \int_{\Omega} G(x, w) d x \\
\geq & \int_{\Omega}\left[\left(c(a, a)-\lambda_{1}(a)\right) a(x)-p \epsilon\right]\left(w^{+}\right)^{p} d x \\
& +\int_{\Omega}(c(a, a) a(x)-\mu b(x)-p \epsilon)\left(w^{-}\right)^{p} d x-p C_{\epsilon}|\Omega| .
\end{aligned}
$$

With the condition $\left(\mathrm{f}_{5}\right)$, we can choose $\epsilon$ small enough such that $\left(c(a, a)-\lambda_{1}(a)\right) a(x)-p \epsilon \geq$ $0, c(a, a) a(x)-\mu b(x)-p \epsilon \geq 0$. Then fix $\epsilon$, and we have $\Phi(w) \geq-p C_{\epsilon}|\Omega|$ where $-p C_{\epsilon}|\Omega|$ is a constant. Obviously (3.13) holds true. 
We suppose that $\Phi$ has only a finite number of critical points. Then, for sufficiently small number $c$, we have by [24], that

$$
C_{1}(\Phi, \infty)=H_{1}\left(E, \Phi^{c}\right) \neq 0
$$

Lemma 3.3 (Theorem 2.1 in [21]) Suppose that conditions $\left(\mathrm{f}_{1}\right)-\left(\mathrm{f}_{4}\right)$ hold, and let zero be an isolated critical point of $\Phi$. Then the Morse critical groups for $\Phi$ at zero are trivial, that is,

$$
C_{q}(\Phi, 0)=0, \quad \text { for all } q
$$

Proof of Theorem 3.1 In view of Lemma 3.1 and Lemma 3.2, we have $C_{1}(\Phi, \infty) \neq 0$, and according to the Morse equality, the functional $\Phi$ has at least one critical point $u$ such that $C_{1}(\Phi, u) \neq 0$. Since $C_{1}(\Phi, 0)=0$ by Lemma 3.3, we conclude that $u \neq 0$. The proof is complete.

Proof of Theorem 3.2 Here we only consider the case $(\lambda, \mu) \in C_{l}^{+}$, since the case $(\lambda, \mu) \in C_{l}^{-}$ can be done similarly. Let $(\lambda, \mu)=\left(s+c_{l}^{+}\left(s, t, s_{0}\right), t+c_{l}^{+}\left(s, t, s_{0}\right)\right)$, where $s_{0}=c_{l}^{+}\left(s, t, s_{0}\right)$. We introduce a new functional as follows:

$$
\begin{aligned}
\Phi_{j}(u)= & \Phi(u)+\frac{1}{j} \int_{\Omega} a|u|^{p} \\
= & \int_{\Omega}|\nabla u|^{p}-s a\left(u^{+}\right)^{p}-\left(t+c_{l}^{+}\left(s, t, s_{0}\right)\right) b\left(u^{-}\right)^{p}+c_{l}^{+}\left(s, t, s_{0}\right) a\left(u^{-}\right)^{p} \\
& -\left(c_{l}^{+}\left(s, t, s_{0}\right)-\frac{1}{j}\right) a|u|^{p}-p G(x, u) .
\end{aligned}
$$

First we will show that, for sufficiently large $j$, there is some $u_{j} \in W_{0}^{1, p}(\Omega)$ such that

$$
\left\|u_{j}\right\|\left\|\Phi_{j}^{\prime}\left(u_{j}\right)\right\| \rightarrow 0, \quad \inf \Phi_{j}\left(u_{j}\right)>-\infty
$$

Let $A^{l-2} \in \mathcal{F}_{l-1}^{+}(a)$ be as in Section 2. By (2.12), there exists $j_{0}$, for any $j \geq j_{0}$, and we have

$$
\max _{u \in A^{l-2}} J_{s, t, s_{0}}^{+}(u) \leq c_{l}^{+}\left(s, t, s_{0}\right)-\frac{2}{j} .
$$

For such $j, u \in A^{l-2}$ and $R>0$, by $\left(\mathrm{F}_{1}\right)$ and $a(x) \geq \epsilon_{0}$ (a.e. in $\Omega$ ) we will have

$$
\begin{aligned}
\Phi_{j}(R u) & =R^{p}\left[J_{s, t, s_{0}}^{+}-\left(c_{l}^{+}\left(s, t, s_{0}\right)-\frac{1}{j}\right)\right]-p \int_{\Omega} G(x, R u) \\
& \leq-\frac{R^{p}}{j}+p\left(\|V\|_{p}^{p-q}\|u\|_{p}^{q} R^{q}+\|W\|_{p}^{p-1}\|u\|_{p} R\right) \\
& \leq-\frac{R^{p}}{j}+C\left(\|V\|_{p}^{p-q} R^{q}+\|W\|_{p}^{p-1} R\right) \\
& \leq-\frac{R^{p}}{j}+C\left(R^{q}+1\right) .
\end{aligned}
$$


Then we see that

$$
\max _{A^{l-2}} \Phi_{j}(R u) \rightarrow-\infty, \quad \text { as } R \rightarrow \infty
$$

Let

$$
F=\left\{u \in W_{0}^{1, p}(\Omega): J_{s, t, s_{0}}^{+} \geq c_{l}^{+}\left(s, t, s_{0}\right) \int_{\Omega} a|u|^{p}\right\} .
$$

For $u \in F$, by (3.18) and $\left(\mathrm{F}_{1}\right)$, we can deduce the following from (3.15):

$$
\begin{aligned}
\Phi_{j}(u) & \geq \frac{\int_{\Omega} a|u|^{p}}{j}-p\left(\|V\|_{p}^{p-q}\|u\|_{p}^{q}+\|W\|_{p}^{p-1}\|u\|_{p}\right) \\
& \geq \frac{\int_{\Omega} a|u|^{p}}{j}-C\left(\|V\|_{p}^{p-q}\left(\int_{\Omega} a|u|^{p}\right)^{\frac{q}{p}}+\|W\|_{p}^{p-1}\left(\int_{\Omega} a|u|^{p}\right)^{\frac{1}{p}}\right) \\
& \geq \frac{\int_{\Omega} a|u|^{p}}{j}-C\left(\left(\int_{\Omega} a|u|^{p}\right)^{\frac{q}{p}}+1\right) .
\end{aligned}
$$

Denote $r:=\left(\int_{\Omega} a|u|^{p}\right)^{\frac{1}{p}}, k(r):=\frac{r^{p}}{j}-C\left(r^{q}+1\right)$. It is sufficient to deduce that $k(r)(r \in[0,+\infty))$ takes its minimum at point $r_{m}=\left(\frac{C q j}{p}\right)^{\frac{1}{p-q}}$, and its minimum is

$$
\max _{r \geq 0} k(r)=k\left(\left(\frac{C q j}{p}\right)^{\frac{1}{p-q}}\right)=\left(\frac{q j}{p}\right)^{\frac{q}{p-q}} C^{\frac{p}{p-q}}\left(\frac{q}{p}-1\right)-C .
$$

Thus, we have

$$
\inf _{u \in F} \Phi_{j}(u) \geq C_{j}:=\min _{r \geq 0} k(r)=\left(\frac{q j}{p}\right)^{\frac{q}{p-q}} C^{\frac{p}{p-q}}\left(\frac{q}{p}-1\right)-C>-\infty .
$$

Now using (3.17), for any $j \geq j_{0}$, we can choose sufficiently high $R_{j}>0$, thus

$$
\max \Phi_{j}(B)<C_{j}
$$

where $B=\left\{R_{j} u: u \in A^{l-2}\right\}$. In fact, (3.20) holds only if $R_{j}$ is larger than the positive solution of the following equation:

$$
-\frac{R^{p}}{j}+C\left(R^{q}+1\right)=C_{j}=\left(\frac{q j}{p}\right)^{\frac{q}{p-q}} C^{\frac{p}{p-q}}\left(\frac{q}{p}-1\right)-C .
$$

It is sufficient to find that (3.20) holds as $R_{j} \geq C j^{\frac{1}{p-q}}$. As a matter of convenience, and without loss of generality, we suppose $R_{j_{0}}$ is large enough and satisfies

$$
R_{j+1}=\frac{1}{\theta} R_{j}, \quad j=j_{0}, j_{0}+1, \ldots
$$

Then we will consider the homotopy-stable family of compact subsets of $W_{0}^{1, p}(\Omega)$ with boundary $B$ given by

$$
\begin{aligned}
\mathcal{F}_{j}^{+}= & \left\{A \subset W_{0}^{1, p}: \text { there is a continuous surjection } h: S_{+}^{l-1} \rightarrow A\right. \\
& \text { such that } \left.\left.h\right|_{S^{l-2}}=R_{j} h_{l-2}\right\},
\end{aligned}
$$


where $h_{l-2}: S^{l-2} \rightarrow A^{l-2}$ is as in Section 2. We claim that the set $F$ is dual to the class $\mathcal{F}_{j}^{+}$, i.e.,

$$
F \cap B=\emptyset, \quad F \cap A \neq \emptyset, \quad \forall A \in \mathcal{F}_{j}^{+} .
$$

It is clear from (3.19) and (3.20) that $F \cap B=\emptyset$. Let $A \in \mathcal{F}_{j}^{+}$. If $0 \in A$, then we are done. Otherwise, we denote by $\pi$ the radial projection onto $S, \pi(A) \in \mathcal{F}_{l}^{+}$, and we have

$$
\max _{u \in \pi(A)} J_{s, t, s_{0}}^{+}(u) \geq c_{l}^{+}\left(s, t, s_{0}\right)
$$

So $F \cap \pi(A) \neq \emptyset$, and we obtain $F \cap A \neq \emptyset$.

Now let us define a minimax sequence on the class $\mathcal{F}^{+}$:

$$
c_{j}:=\inf _{A \in \mathcal{F}_{j}^{+}} \max _{u \in A} \Phi_{j}(u)
$$

Noting the truth that the set $F$ is dual to the class $\mathcal{F}_{j}^{+}$, and by (3.19), we have

$$
c_{j} \geq C_{j}>\max _{u \in R_{j} A^{l-2}} \Phi_{j}(u) .
$$

Hence it follows from a deformation argument of Cerami [22] that, for the above $j$, there exists a sequence $u_{j}$ such that

$$
\left\|u_{j}\right\|\left\|\Phi_{j}^{\prime}\left(u_{j}\right)\right\|<\frac{1}{j}, \quad\left|\Phi_{j}\left(u_{j}\right)-c_{j}\right|<\frac{1}{j} .
$$

It is easy to prove that $\left\{c_{j}\right\}_{j_{0}}^{\infty}$ is a non-decreasing sequence. According to (3.24) and (3.25), for $j \geq j_{0}$ we can get

$$
\begin{aligned}
c_{j 0}+\frac{1}{j_{0}} & \geq c_{j}+\frac{1}{j} \\
& \geq \Phi_{j}\left(u_{j}\right) \\
& \geq c_{j}-\frac{1}{j} \\
& \geq C_{j}-\frac{1}{j} \\
& =\left(\frac{q j}{p}\right)^{\frac{q}{p-q}} C^{\frac{p}{p-q}}\left(\frac{q}{p}-1\right)-C .
\end{aligned}
$$

We claim that the sequence $\left\{c_{j}\right\}_{j_{0}}^{\infty}$ is bounded. Assume the contrary, that is, $c_{j} \rightarrow-\infty$ as $j \rightarrow \infty$, and then we will prove that $\left\{c_{j}\right\}_{j_{0}}^{\infty}$ has some fixed growth rate, which contradicts (3.26).

In fact, for a fixed number $j \geq j_{0}$ and $\forall \epsilon>0$, there exists $A \in \mathcal{F}_{j}^{+}$and a continuous surjection $h$, such that $A=h\left(S_{+}^{l-1}\right)$ and

$$
\max _{u \in S_{+}^{L^{-1}}} \Phi_{j}(h(u))<c_{j}+\epsilon .
$$


Noting that $H(\cdot):=\frac{R_{j+1}}{R_{j}} h(\cdot)=\theta^{-1} h(\cdot) \in \mathcal{F}_{j+1}^{+}$. Therefore, for all $u \in S_{+}^{l-1}$, we have

$$
\begin{aligned}
c_{j}+\epsilon> & \Phi_{j}(h(u))=\Phi(h(u))+\frac{1}{j} \int_{\Omega} a|h(u)|^{p} \\
= & \int_{\Omega}|\nabla h(u)|^{p}-\left(s+c_{l}^{+}\left(s, t, s_{0}\right)\right)\left(h(u)^{+}\right)^{p}-\left(t+c_{l}^{+}\left(s, t, s_{0}\right)\right)\left(h(u)^{-}\right)^{p} \\
& -p G(x, h(u))+\frac{1}{j} \int_{\Omega} a|h(u)|^{p} \\
= & \left(\frac{R_{j}}{R_{j+1}}\right)^{p}\left(\int_{\Omega}|\nabla H(u)|^{p}-\left(s+c_{l}^{+}\left(s, t, s_{0}\right)\right)\left(H(u)^{+}\right)^{p}-\left(t+c_{l}^{+}\left(s, t, s_{0}\right)\right)\left(H(u)^{-}\right)^{p}\right. \\
& \left.-p G(x, H(u))+\frac{1}{j+1} \int_{\Omega} a|H(u)|^{p}\right) \\
& +\left(\frac{R_{j}}{R_{j+1}}\right)^{p} \int_{\Omega} p G(x, H(u))-\int_{\Omega} p G(x, h(u))+\frac{1}{j(j+1)} \int_{\Omega} a|h(u)|^{p} \\
= & \theta^{p} \Phi_{j+1}(H(u))+\theta^{p} \int_{\Omega} p G\left(x, \frac{h(u)}{\theta}\right)-\int_{\Omega} p G(x, h(u))+\frac{1}{j(j+1)} \int_{\Omega} a|h(u)|^{p} \\
\geq & \theta^{p} \Phi_{j+1}(H(u))-C_{M}, \quad
\end{aligned}
$$

where $C_{M}$ is a constant and only related to the condition $\left(\mathrm{F}_{2}\right)$. Noting the arbitrariness of $u \in S_{+}^{l-1}$, we have

$$
\begin{aligned}
c_{j}+\epsilon & \geq \theta^{p} \max _{u \in S_{+}^{l-1}} \Phi_{j+1}(H(u))-C_{M} \\
& \geq \theta^{p} c_{j+1}-C_{M} .
\end{aligned}
$$

According to the arbitrariness of $\epsilon$, we have

$$
c_{j} \geq \theta^{p} c_{j+1}-C_{M} .
$$

From the assumption that $c_{j} \rightarrow-\infty$ as $j \rightarrow \infty$, there will exist $j_{1} \geq j_{0}$, such that $c_{j_{1}}<0$, and then we can get

$$
\left|c_{j}\right| \leq \theta^{p}\left|c_{j+1}\right|+C_{M}, \quad j=j_{1}, j_{1}+1, \ldots
$$

Suppose that $\left|c_{j}\right|$ is large enough, and then we can translate the last formula into the following:

$$
\left|c_{j}\right| \leq \theta\left|c_{j+1}\right|, \quad j=j_{1}, j_{1}+1, \ldots
$$

Then we have

$$
\left|c_{j+1}\right| \geq \theta^{j_{1}-j-1}\left|c_{j_{1}}\right|, \quad j=j_{1}, j_{1}+1, \ldots,
$$

contradicting (3.26). Thus, $\left\{c_{j}\right\}_{j_{0}}^{\infty}$ is bounded. According to (3.25), we obtain (3.16). 
We complete the proof by showing that a subsequence of $\left\{u_{j}\right\}$ mentioned in (3.16) converges to a solution to (1.1). It is sufficient to prove that $\left\{u_{j}\right\}$ is bounded. Assume the contrary, that is, $\left\|u_{j}\right\| \rightarrow \infty$. Setting $v_{j}:=\frac{u_{j}}{\left\|u_{j}\right\|}$, then up to a subsequence, we still denote the subsequence by $\left\{v_{j}\right\}$. There is a $v_{0}$ such that $v_{j} \rightarrow v_{0}$ weakly in $W_{0}^{1, p}(\Omega)$, strongly in $L^{p}(\Omega)$, and almost everywhere in $\Omega$. We have

$$
\begin{aligned}
\int_{\Omega}\left|\nabla v_{j}\right|^{p-2} \nabla v_{j} \nabla\left(v_{j}-v_{0}\right)= & \frac{\left(\Phi_{j}^{\prime}\left(u_{j}\right), v_{j}-v_{0}\right)}{p\left\|u_{j}\right\|_{1, p}^{p-1}}+\int_{\Omega}\left[\left(\lambda-\frac{1}{j}\right) a\left(v_{j}^{+}\right)^{p-1}\right. \\
& \left.+\left(\mu b-\frac{1}{j} a\right)\left(v_{j}^{-}\right)^{p-1}-\frac{g\left(x, u_{j}\right)}{\left\|u_{j}\right\|_{1, p}^{p-1}}\right]\left(v_{j}-v_{0}\right) \rightarrow 0 .
\end{aligned}
$$

It is sufficient to obtain

$$
\int_{\Omega}\left|\nabla v_{j}\right|^{p-2} \nabla v_{j} \nabla\left(v_{j}-v_{0}\right) d x \rightarrow 0, \quad j \rightarrow \infty
$$

Hence, we can deduce that $v_{j} \rightarrow v_{0}$ strongly in $W_{0}^{1, p}$, and $\left\|v_{0}\right\|=1$. For each $w \in W_{0}^{1, p}$, we have

$$
\begin{aligned}
\frac{\left(\Phi_{j}^{\prime}\left(u_{j}\right), w\right)}{p\left\|u_{j}\right\|_{1, p}^{p-1}}= & \int_{\Omega}\left|\nabla v_{j}\right|^{p-2} \nabla v_{j} \nabla w-\int_{\Omega}\left[\left(\lambda-\frac{1}{j}\right) a\left(v_{j}^{+}\right)^{p-1}\right. \\
& \left.+\left(\mu b-\frac{1}{j} a\right)\left(v_{j}^{-}\right)^{p-1}-\frac{g\left(x, u_{j}\right)}{\left\|u_{j}\right\|_{1, p}^{p-1}}\right] w .
\end{aligned}
$$

Going to the limit as $j \rightarrow \infty$, we get

$$
\int_{\Omega}\left|\nabla v_{0}\right|^{p-2} \nabla v_{0} \nabla w-\left[\lambda a\left(v_{0}^{-}\right)^{p-1}-\mu b\left(v_{0}^{+}\right)^{p-1}\right] w=0 .
$$

So $v_{0} \in E_{\lambda a, \mu b}$. Thus,

$$
\frac{\left(\Phi_{j}^{\prime}\left(u_{j}\right), u_{j}\right)}{p}-\Phi_{j}\left(u_{j}\right)=\int_{\Omega}\left[p G\left(x, u_{j}\right)-g\left(x, u_{j}\right) u_{j}\right] \rightarrow+\infty
$$

contradicting (3.16). Hence, $\left\{u_{j}\right\}$ is bounded. There exists a subsequence of $\left\{u_{j}\right\}$ converging to a weak solution to (1.1).

\section{Competing interests}

The authors declare that they have no competing interests.

\section{Authors' contributions}

All authors contributed equally to the writing of this paper. The authors read and approved the final manuscript.

\section{Author details}

'Department of Mathematics, Foshan University, Foshan, Guangdong 528000, P.R. China. ${ }^{2}$ School of Mathematical Sciences, South China Normal University, Guangzhou, Guangdong 510631, P.R. China.

\section{Acknowledgements}

This research was supported in part by the Natural Science Foundation of China under grants 11501106 and 11226303 , the Natural Science Foundation of Guangdong Province of China under grant 2016A030313835, the Excellent Teacher Scheme in Guangdong Higher Education Institutions under grant YQ2014332 and the Training Programme Foundation for Excellent Young Scholar of Guangdong Province under grant YQ2015164. 


\section{Publisher's Note}

Springer Nature remains neutral with regard to jurisdictional claims in published maps and institutional affiliations.

Received: 2 December 2016 Accepted: 2 March 2017 Published online: 14 March 2017

\section{References}

1. Fučik, S: Solvability of Nonlinear Equations and Boundary Value Problems. Springer, Dordrecht (1980)

2. Dancer, N: On the Dirichlet problem for weakly nonlinear elliptic partial differential equation. Proc. R. Soc. Edinb. 76 , 283-300 (1977)

3. Arias, M, Campos, J: Radial Fučik spectrum of the Laplace operator. J. Math. Anal. Appl. 156, 654-666 (1995)

4. Dancer, N: Generic domain dependance for nonsmooth equations and the open set problem for jumping nonlinearities. Topol. Methods Nonlinear Anal. 1, 139-150 (1993)

5. Micheletti, AM: A remark on the resonance set for a semilinear elliptic equation. Proc. R. Soc. Edinb. 124, 803-809 (1994)

6. Drábek, P: Solvability and Bifurcations of Nonlinear Equations. Pitman Research Notes in Mathematics, vol. 264. Wiley, New York (1992)

7. Arias, J, Campos, M: Fučik spectrum of a singular Sturm-Liouville problem. Nonlinear Anal. TMA 27, 679-697 (1996)

8. Rynne, B: The Fučik spectrum of general Sturm-Liouville problems. J. Differ. Equ. 161, 87-109 (2000)

9. Reichel, W, Walter, W: Sturm-Liouville type problems for the $p$-Laplacian under asymptotic nonresonance conditions. J. Differ. Equ. 156, 50-70 (1999)

10. Alif, M, Gossez, J-P: On the Fučik spectrum with indefinite weights. Differ. Integral Equ. 14, 1511-1530 (2001)

11. Lindqvist, P: On the equation $\operatorname{div}\left(|\nabla u|^{p-2} \nabla u\right)+\lambda|u|^{p-2} u=0$. Proc. Am. Math. Soc. 109(1), 157-164 (1990); Addendum: Proc. Am. Math. Soc. 116(2), 583-584 (1992)

12. Cuesta, M: Eigenvalue problems for the $p$-Laplacian with indefinite weight. Electron. J. Differ. Equ. 2001, 33 (2001)

13. Arias, M, Campos, J, Cuesta, M, Gossez, J-P: Asymmetric elliptic problems with indefinite weights. Ann. Inst. Henri Poincaré, Anal. Non Linéaire 19, $581-616$ (2002)

14. Cuesta, M, De Figueiredo, D, Gossez, JP: The beginning of the Fučik spectrum of the $p$-Laplacian. J. Differ. Equ. 159, 212-238 (1999)

15. Micheletti, A, Pistoia, A: On the Fučik spectrum for the p-Laplacian. Differ. Integral Equ. 14, 867-882 (2001)

16. Perera, K: Resonance problems with respect to the Fučik spectrum of the $p$-Laplacian. Electron. J. Differ. Equ. 2002, 36 (2002)

17. Perera, K, Squassina, $M$, Yang, Y: A note on the Dancer-Fučik spectra of the fractional $p$-Laplacian and Laplacian operators. Adv. Nonlinear Anal. 4(1), 13-23 (2015)

18. Pucci, P, Radulescu, V: The impact of the mountain pass theory in nonlinear analysis: a mathematical survey. Boll. Unione Mat. Ital. 9(3), 543-582 (2010)

19. Pucci, P, Radulescu, V: Combined effects in quasilinear elliptic problems with lack of compactness. Atti Accad. Naz. Lincei, Rend. Lincei, Mat. Appl. 22(2), 189-205 (2011)

20. Barbu, L, Enache, C: Maximum principles, Liouville-type theorems and symmetry results for a general class of quasilinear anisotropic equations. Adv. Nonlinear Anal. 5(4), 395-405 (2016)

21. Guo, Y, Liu, J: Solutions of $p$-sublinear $p$-Laplacian equation via Morse theory. J. Lond. Math. Soc. 72, 632-644 (2005)

22. Cerami, G: An existence criterion for the critical points on unbounded manifolds. Istit. Lomb. Accad. Sci. Lett. Rend. A. 112(2), 332-336 (1979)

23. Bartolo, P, Benci, V, Fortunato, D: Abstract critical point theorems and applications to nonlinear problems with strong resonance at infinity. Nonlinear Anal. 7, 981-1012 (1983)

24. Bartsch, T, Li, SJ: Critical point theory for asymptotically quadratic functionals and applications to problems with resonance. Nonlinear Anal. 28, 419-441 (1997)

25. Drábek, P, Robinson, S: Resonance problems for the $p$-Laplacian. J. Funct. Anal. 169, 189-200 (1999)

26. Ghoussoub, M: Duality and Perturbation Methods in Critical Point Theory. Cambridge University Press, Cambridge (1993)

27. Chang, KC: Infinite Dimensional Morse Theory and Multiple Solutions of Nonlinear Differential Equations. Birkhäuser, Boston (1993)

28. Anane, A, Tsouli, N: On the second eigenvalue of the $p$-Laplacian. In: Benkirane, A, Gossez, J-P (eds.) Nonlinear Partial Differential Equations. Pitman Research, Notes in Math., vol. 343, pp. 1-9 (1996)

\section{Submit your manuscript to a SpringerOpen ${ }^{\circ}$ journal and benefit from:}

- Convenient online submission

Rigorous peer review

- Immediate publication on acceptance

- Open access: articles freely available online

- High visibility within the field

- Retaining the copyright to your article 